\title{
Genetische Analyse der Population des Hinterwälder Rindes
}

\begin{abstract}
Title of the paper: Genetic analysis of the population of Hinterwald cattle

Since 1994 the Hinterwald population is devided into one of dairy cows and one of suckling cows. The bulls are available to both subpopulations.

By means of herdbook records of the year of 2002 there were gained 79 bulls, 698 dairy cows and 517 suckling cows. Considering five generations of ancestors the subpopulations have been investigated with regard to genetic contribution, generation intervals, and circumstances of relationship and inbreeding.

The average percentage of Hinterwald blood in the current population amounts to $97 \%$. The genetic portions of other breeds are low and mostly originating from Vorderwald cattle. The mean generation interval (parentscurrent animals) is about 5 years. Between the sires and current animals it is shorter (about 4 years) than between the dams and the current animals (about 7 years). The average coefficient of relationship among the bulls is the highest (3.7\%) compared to the dairy cows (2.2\%) and the suckling cows $(0.9 \%)$. The mean degree of inbreeding is 1.2 up to $1.3 \%$, and $37 \%$ of the bulls, $57 \%$ of the dairy cows and $74 \%$ of the suckling cows are not inbred. The increase of inbreeding is rather low and doesn't surmount $0.7 \%$ in all subpopulations. Therefore it can be assumed that the Hinterwald cattle at present is not subjected to any danger of inbreeding.
\end{abstract}

Key Words: Hinterwald, gene contribution, generation interval, degree of relationship, coefficient of inbreeding, rate of inbreeding

\section{Zusammenfassung}

Das Hinterwälder Rind wird seit 1994 in Form einer Milchvieh- und einer Mutterkuhpopulation gezüchtet. Beiden Teilpopulationen steht ein und derselbe Bullenbestand zur Verfügung.

Anhand von Zuchtbuchunterlagen des Jahres 2002 wurden 79 Bullen, 698 Milchkühe und 517 Mutterkühe einschließlich ihrer Vorfahren erfasst. Unter Berücksichtigung von fünf Ahnengenerationen wurden die Teilpopulationen hinsichtlich ihrer Genanteile, des Generationsintervalls sowie der Verwandtschafts- und Inzuchtverhältnisse untersucht.

Im Durchschnitt lässt sich bei den Tieren ein von den Hinterwäldern stammender Genanteil von ca. 97 \% nachweisen. Fremdgenanteile sind gering und rühren vorrangig von der Vorderwälder Rasse her. Das mittlere Generationsintervall (Eltern-Probanden) beträgt ca. 5 Jahre, wobei es mit dem Pfad Väter-Probanden einen kürzeren Zeitraum einnimmt (ca. 4 Jahre) als hinsichtlich des Pfades Mütter-Probanden (ca. 7 Jahre). Der mittlere Verwandtschaftskoeffizient erweist sich innerhalb der Bullen am höchsten (3,7 \%). Für die Milchkühe wurde er mit 2,2 \%, für die Mutterkühe mit nur 0,9 \% festgestellt. Die durchschnittliche Inzucht beträgt 1,2-1,3 \%, wobei 37 \% der Bullen, 53 \% der Milchkühe und 74 \% der Mutterkühe nicht ingezogen sind. Die Inzuchtrate ist durchweg gering und liegt in allen Teilpopulationen unter $0,7 \%$. Es kann somit unterstellt werden, dass das Hinterwälder Rind derzeit keinen akuten Gefahren seitens der Inzucht ausgesetzt ist.

Schlüsselwörter: Hinterwälder, Genanteile, Generationsintervall, Verwandtschaft, Inzuchtkoeffizient, Inzuchtrate

1.

\section{Einleitung}

Wenngleich die Herkunft des Hinterwälder Rindes auch nicht eindeutig geklärt ist, dürfte es sich bei ihm um eine sehr alte Rasse handeln, die sich im Schwarzwald etabliert hat und als ein Kleinod der deutschen Tierzucht angesehen werden kann. Um so bedauerlicher mutet es an, dass bereits in den 1960er Jahren Diskussionen um seinen 
Fortbestand geführt werden mussten. Neben diversen Fördermaßnahmen entschied man sich in den 1970er Jahren für einen Einkreuzungsversuch mit Fleckvieh und Vorderwäldern, um eine Verbesserung der Fleischleistung vor allem für die Mutterkuhhaltung zu erzielen (KÖTTERITSZCH und MAUS, 1999). Aufgrund der zunehmenden Konzentration der Population auf eine Linie beschlossen die Zuchtverantwortlichen zudem, mit kleinrahmigen Vorderwälder Bullen eine „Auffrischung“ vorzunehmen; die Maßnahme nahm 1972 ihren Anfang. Die Mutterkuhhaltung nahm seitdem an Bedeutung zu und bot sich zunehmend für vermehrt arbeitsextensiv ausgerichtete Nebenerwerbsbetriebe an. Aufgrund unterschiedlicher züchterischer Ansprüche an Milch- und Mutterkühe, kam der zuständige Zuchtverband den Mutterkuhhaltern entgegen, um sie von einer völligen Aufgabe der für den Schwarzwald höchst bedeutsamen Rinderhaltung abzuhalten; er entschloss sich 1994, die Population der Hinterwälder in zwei Teilpopulationen zu gliedern (MAUS, 1997). Um allerdings eine totale Spaltung der Rasse zu vermeiden, wurde von einer Differenzierung der Bullen abgesehen.

Somit darf gehofft werden, dass mit dieser und anderen Maßnahmen die Erhaltung der kleinen Population der Hinterwälder Rinder gesichert bleibt. Sie sind nicht nur optimal an die natürlichen Bedingungen des Schwarzwaldes angepasst und damit in der Lage, dessen Landschaftsbild zu bewahren, sondern verkörpern als altes bäuerliches Kulturgut mit langer Tradition ein Markenzeichen einer Region und bilden somit nicht zuletzt auch eine touristische Attraktion ihres Verbreitungsgebietes.

Die vorliegende Analyse macht sich zur Aufgabe, die aktuelle Situation der Hinterwälder Rinder in verschiedener Hinsicht zu untersuchen. Angesichts früherer Einkreuzung von Fremdrassen interessiert die genetische Zusammensetzung der Rasse. Auch wenn derzeit die Zahl der Herdbuchkühe ca. 1200 beträgt, darf nicht übersehen werden, dass der Bestand an Herdbuchkühen in jüngerer Vergangenheit mitunter nur ca. 250 Tiere umfasste, so dass den Inzucht- und Verwandtschaftsverhältnissen in der Population zudem eine besondere Bedeutung zukommt.

Als Datengrundlage dienten die Zuchtbuchauszüge der Mitte 2002 aktiven Herdbuchtiere des Hinterwälder Rindes. Bei den Bullen handelte es sich neben den lebenden Tieren auch um solche, die aufgrund ihrer Spermareserven an der Zucht beteiligt sind. Insgesamt wurden 1294 Zuchttiere sowie, soweit bekannt, deren Vorfahren erfasst. Im Einzelnen handelt es sich um 79 Bullen, 698 Milchkühe und 517 Mutterkühe.

Die Analyse der Population wurde für die beiden Teilpopulationen getrennt vorgenommen, wobei die Bullen sowohl der Milchvieh- als auch der Mutterkuhpopulation zugeordnet werden mussten. Sämtliche Berechnungen erfolgten mit Hilfe des Programms OPTI-MATE Vers. 3.3 von SCHMIDT (2000). Wegen der Lücken in älteren Ahnengenerationen wurden in sämtlichen Teiluntersuchungen nur fünf Vorfahrengenerationen berücksichtigt, womit sich zusätzlich eine gute Vergleichbarkeit mit Ergebnissen aus früheren entsprechenden Untersuchungen verbindet.

Die Genanteile fremder Populationen wurden für jeden Probanden anhand der Rassezugehörigkeit der Vorfahren in der 5. Ahnengeneration bestimmt. Daraus konnten die durchschnittlichen Genanteile der gesamten Population ermittelt werden.

Generationsintervalle bzw. partielle Generationsintervalle wurden als durchschnittliches Alter der jeweiligen Eltern bei Geburt ihrer Nachkommen berechnet. In Fällen, in 
denen sich die Angaben zur Geburt auf das Geburtsjahr beschränkten, wurde der 01.07. als Geburtstag eingesetzt, um Fehler zu minimieren.

Die Schätzung von Inzucht- und Verwandtschaftskoeffizienten wurde nach der von WRIGHT (1923) eingeführten Pedigree-Methode vorgenommen. Die entsprechenden Populationsparameter entstanden aus der Mittelung sämtlicher Einzelkoeffizienten. In vergleichbarer Weise erfolgte die Ermittlung der Verwandtschaft zwischen den Kühen und den Bullen, indem dieser die Schätzung der Verwandtschaftskoeffizienten aller männlichen mit sämtlichen weiblichen Zuchttieren voranging.

Trotz Beschränkung auf fünf Ahnengenerationen stellten sich die Stammbäume der Tiere mitunter als unvollständig heraus, womit Fehleinschätzungen der Inzuchtkoeffizienten eintreten würden, weshalb PIRCHNER (2002) auf die Grenzen der PedigreeAnalyse hinweist. Um jedoch Fehler auf ein Mindestmaß zu verringern, wurde ein Vollständigkeitsindex, der von SCHMIDT (2000) näher beschrieben wird, zur Korrektur der Inzuchtkoeffizienten angewandt. Der Vollständigkeitsindex gibt den Anteil tatsächlich besetzter Inzuchtbindungsstellen von Pedigrees an der Gesamtzahl möglicher Bindungen an (SCHMIDT et al., 1993).

Die Inzuchtrate $\Delta \mathrm{F}$ wurde nach unterschiedlichen Vorgehensweisen geschätzt:

$\Delta \mathrm{F}_{1} \quad$ Mittlere Inzuchtrate pro Generation seit der 5. Ahnengeneration:

$$
\begin{aligned}
& \Delta \mathrm{F}_{1}=\frac{\mathrm{F}_{\mathrm{t}}}{\mathrm{n}-1} \mathrm{~F}_{\mathrm{t}} \begin{array}{l}
= \\
\text { durchschnittlicher Inzuchtkoeffizient der aktuellen } \\
\text { Population }
\end{array} \\
& \mathrm{n} \quad=\text { Anzahl berücksichtigter Ahnengenerationen }
\end{aligned}
$$

$\Delta \mathrm{F}_{2} \quad$ Wie $\Delta \mathrm{F}_{1}$, aber unter Berücksichtigung der Pedigreevollständigkeit

$\Delta \mathrm{F}_{3} \quad$ Inzuchtrate in der letzten Generation:

$$
\Delta \mathrm{F}_{3}=\frac{\mathrm{F}_{\mathrm{t}}-\mathrm{F}_{\mathrm{t}-1}}{1-\mathrm{F}_{\mathrm{t}-1}} \quad \begin{array}{ll}
\mathrm{F}_{\mathrm{t}} & =\text { durchschnittlicher Inzuchtkoeffizient der aktuellen } \\
& \text { Population }
\end{array}
$$

$\Delta \mathrm{F}_{4} \quad$ Wie $\Delta \mathrm{F}_{3}$, aber unter Berücksichtigung der Pedigreevollständigkeit

$\Delta \mathrm{F}_{5} \quad$ Erwartete Inzuchtrate aufgrund der effektiven Anzahl Väter und Mütter:

$$
\Delta \mathrm{F}_{5}=\frac{1}{8 \cdot \mathrm{M}_{\mathrm{e}}}+\frac{1}{8 \cdot \mathrm{W}_{\mathrm{e}}} \quad \begin{array}{ll}
\mathrm{M}_{\mathrm{e}} & =\text { effektive Anzahl männlicher Zuchttiere } \\
\mathrm{W}_{\mathrm{e}} & =\text { effektive Anzahl weiblicher Zuchttiere }
\end{array}
$$

Tabelle 1

Genanteile der Herkunftsrassen (\%) innerhalb der aktuellen Population (Gene contribution of different breeds (per cent) within the current population)

\begin{tabular}{lccccc}
\hline Rasse & Milchkühe & Mutterkühe & Bullen & $\begin{array}{c}\text { Milchvieh- } \\
\text { population }\end{array}$ & $\begin{array}{c}\text { Mutterkuh- } \\
\text { population }^{2)}\end{array}$ \\
\hline Hinterwälder & 63,35 & 41,51 & 73,34 & 64,36 & 44,50 \\
Vorderwälder & 1,59 & 0,89 & 2,29 & 1,66 & 1,07 \\
Schwarzbunte & 0,09 & 0,06 & 0,16 & 0,09 & 0,07 \\
unbekannt & 34,97 & 57,54 & 24,21 & 33,88 & 54,36 \\
\hline${ }^{1)}$ Milchkühe + Bullen ${ }^{2)}$ Mutterkühe + Bullen & & & &
\end{tabular}

${ }^{1)}$ Milchkühe + Bullen; ${ }^{2)}$ Mutterkühe + Bullen

3. $\quad$ Ergebnisse

3.1. Genanteile fremder Populationen

Nachgewiesene Fremdgenanteile stammen von Vorderwäldern und Schwarzbunten, wobei erstere infolge der in den 1970er Jahren vorgenommenen Linienerweiterung den 
größeren Einfluss ausüben (Tab. 1). Allerdings nehmen die Genanteile unbekannter Herkunft einen beträchtlichen Umfang ein, der bei den Mutterkühen erheblich größer ausfällt als bei den Milchkühen und den Bullen. Unterstellt man, dass die Genanteile innerhalb der unbekannten Herkünfte jener der bekannten Herkünfte entsprechen, so resultiert die in Tabelle 2 angegebene Verteilung der Herkunftsrassen.

Tabelle 2

Genanteile der Herkunftsrassen (\%) innerhalb der aktuellen Population unter Vernachlässigung der unbekannten Ahnen (Gene contribution of different breeds (per cent) within the current population neglecting unknown ancestors)

\begin{tabular}{lrrrrr}
\hline Rasse & Milchkühe & Mutterkühe & Bullen & $\begin{array}{c}\text { Milchvieh- } \\
\text { population }\end{array}$ & $\begin{array}{c}\text { Mutterkuh- } \\
\text { population }\end{array}$ \\
\hline Hinterwälder & 97,42 & 97,77 & 96,77 & 97,34 & 97,50 \\
Vorderwälder & 2,44 & 2,09 & 3,02 & 2,51 & 2,34 \\
Schwarzbunte & 0,13 & 0,14 & 0,22 & 0,14 & 0,16 \\
Rotbunte & 0,01 & 0,00 & 0,00 & 0,01 & 0,00 \\
\hline
\end{tabular}

Aufgrund dieser Korrektur würde sich die Population insgesamt durch hohe Rassereinheit auszeichnen; die Fremdgenanteile betragen demzufolge lediglich ca. $3 \%$ bei den Bullen und ca. 2,5 \% bei den Kühen. Zudem macht sich ein sehr geringer Einfluss der Rotbunten bemerkbar.

\subsection{Generationsintervalle}

Tabelle 3 gibt zu erkennen, dass die Generationsintervalle Väter-Probanden um zwei bis über drei Jahre länger ausfallen als die Generationsintervalle Mütter-Probanden. Dieser Unterschied tritt bei den Generationsintervallen hinsichtlich der Pfade der Väter bzw. Mütter zu den Bullen besonders deutlich hervor.

Abbildung 1 verdeutlicht, dass sich die Generationsintervalle während der zurückliegenden Generationen verringert haben. Eine geringe Ausnahme machen die Bullen, bei denen sich in der letzten Generation (Eltern-Probanden) ein Anstieg bemerkbar macht. Ergänzend wird in Abbildung 2 die Entwicklung der Generationsintervalle innerhalb der männlichen und weiblichen Vorfahren der Bullen dargestellt. Daraus wird ersichtlich, dass oben besagter Anstieg vorrangig bei den weiblichen Vorfahren der Bullen zu suchen ist.

Tabelle 3

Mittlere Generationsintervalle (Jahre) (Mean generation intervals (years))

\begin{tabular}{lccccc}
\hline Pfad & Milchkühe & Mutterkühe & Bullen & $\begin{array}{c}\text { Milchvieh- } \\
\text { population }\end{array}$ & $\begin{array}{c}\text { Mutterkuh- } \\
\text { population }\end{array}$ \\
\hline Eltern - Probanden & 5,01 & & 5,75 & 5,09 & 4,78 \\
& $(1,86-16,55)$ & $(1,51-18,58)$ & $(2,14-13,11)$ & $(1,86-16,55)$ & $(1,51-18,58)$ \\
Väter - Probanden & 3,53 & 3,68 & 3,37 & 3,51 & 3,62 \\
& $(1,65-16,86)$ & $(1,49-19,92)$ & $(1,82-10,07)$ & $(1,65-16,86)$ & $(1,49-19,92)$ \\
Mütter - Probanden & 6,49 & 5,82 & 6,81 & 6,53 & 5,94 \\
& $(2,07-16,24)$ & $(1,53-15,21)$ & $(2,46-16,15)$ & $(2,07-16,15)$ & $(1,53-16,15)$ \\
\hline
\end{tabular}

\subsection{Inzucht}

3.3.1. Verwandtschaft innerhalb der Population

Gepaart mit der höchsten Vollständigkeit ihrer Pedigrees, erweist sich die mittlere Verwandtschaft innerhalb der Bullen, verglichen mit den weiblichen Teilpopulationen als deutlich enger (Tab. 4). 


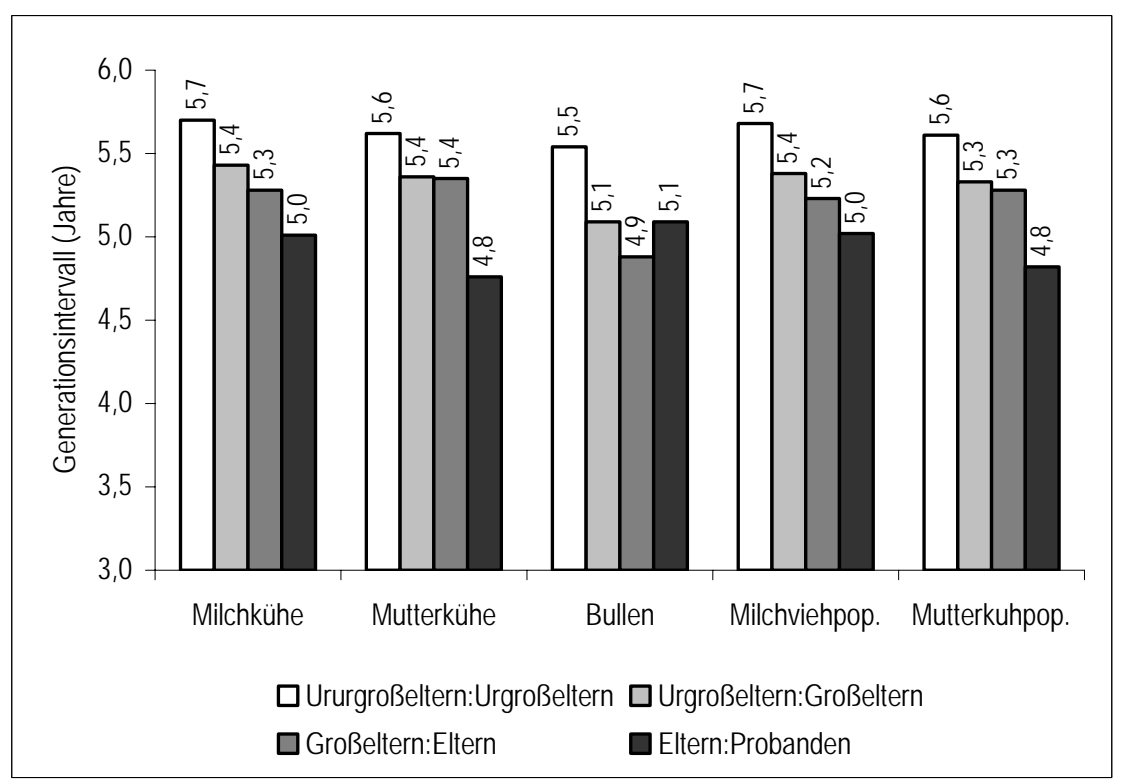

Abb. 1: Entwicklung der Generationsintervalle (Development of the generation intervals)

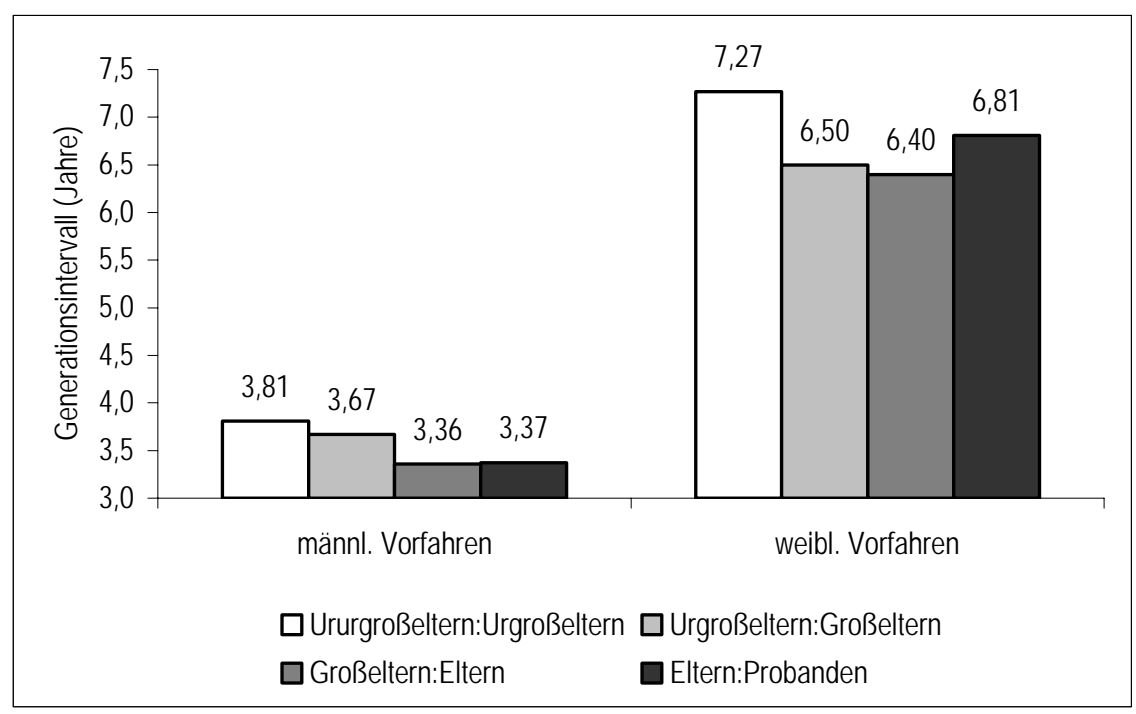

Abb. 2: Entwicklung der Generationsintervalle innerhalb der männlichen und weiblichen Vorfahren der Bullen (Development of the generation intervals within the male and female ancestors of the steers)

Zudem lässt sich innerhalb der Milchkühe eine wesentlich höhere durchschnittliche Verwandtschaft feststellen als bei den Mutterkühen. Allerdings trifft für letztere ein sehr geringer Vollständigkeitsindex zu. Trotz der relativ geringen durchschnittlichen Verwandtschaft der Mutterkühe wurde bei ihnen der höchste maximale Wert mit über $75 \%$ gefunden. Besagte Unterschiede spiegeln sich zudem in den Verteilungen der Verwandtschaftskoeffizienten wider, indem innerhalb der Mutterkühe über $60 \%$ der Paare frei von Verwandtschaft sind, wohingegen innerhalb der Milchkühe und der Bullen bei mehr als $70 \%$ der Bindungen die Verwandtschaft bis zu $10 \%$ beträgt. Im Durchschnitt lassen sich zwischen den Bullen und Milchkühen engere verwandtschaftliche Beziehungen nachweisen als dies für die Mutterkühe zutrifft (Tab. 5). Dementsprechend existiert zwischen ihnen und den Vatertieren die doppelte Anzahl Tierpaare ohne jegliche verwandtschaftliche Bindung. 
Tabelle 4

Verwandtschaftskoeffizienten (oben) und deren Verteilung (unten; in \%) (Degrees of relationship (above) and the distribution of them (below; per cent))

\begin{tabular}{cccrrr}
\hline Verwandtschaft & Milchkühe & Mutterkühe & Bullen & $\begin{array}{r}\text { Milchvieh- } \\
\text { population }\end{array}$ & $\begin{array}{c}\text { Mutterkuh- } \\
\text { population }\end{array}$ \\
\hline Mittlerer Verwandtschaftskoeff. $\overline{\mathrm{R}}(\%)$ & 2,16 & 0,89 & 3,68 & 2,25 & 1,05 \\
Mittlerer Vollständigkeitsindex $\overline{\mathrm{VI}}(\%)$ & 54,73 & 25,14 & 75,57 & 56,70 & 29,18 \\
Max. Verwandtschaftskoeff. $\mathrm{R}_{\max }(\%)$ & 68,75 & 75,39 & 55,08 & 68,75 & 75,39 \\
\hline $\mathrm{R}=0$ & 23,30 & 61,57 & 19,57 & 28,10 & 57,16 \\
$0<\mathrm{R} \leq 10$ & 73,73 & 37,04 & 72,09 & 68,59 & 41,14 \\
$10<\mathrm{R} \leq 20$ & 1,94 & 0,84 & 5,13 & 2,23 & 1,08 \\
$20<\mathrm{R} \leq 30$ & 0,82 & 0,43 & 2,34 & 0,84 & 0,46 \\
$30 \mathrm{R} \leq 40$ & 0,12 & 0,03 & 0,29 & 0,11 & 0,04 \\
$40<\mathrm{R} \leq 50$ & 0,03 & 0,06 & 0,06 & 0,04 & 0,06 \\
$50 \mathrm{R}$ & 0,07 & 0,03 & 0,52 & 0,09 & 0,05 \\
\hline
\end{tabular}

Tabelle 5

Durchschnittliche Verwandtschaft zwischen Bullen und Kühen (Mean relationship between the bulls and the cows)

\begin{tabular}{|c|c|c|}
\hline Verwandtschaft & Milchkühe & Mutterkühe \\
\hline Mittlerer Verwandtschaftskoeffizient $\overline{\mathrm{R}}$ (\%) & 2,60 & 1,42 \\
\hline Mittlerer Vollständigkeitsindex $\overline{\mathrm{VI}}(\%)$ & 64,33 & 39,83 \\
\hline Maximaler Verwandtschaftskoeffizient $\mathrm{R}_{\max }(\%)$ & 56,35 & 75,39 \\
\hline $\mathrm{R}=0$ & 22,82 & 44,59 \\
\hline $0<\mathrm{R} \leq 10$ & 73,74 & 53,15 \\
\hline $10<\mathrm{R} \leq 20$ & 3,28 & 1,64 \\
\hline $20<\mathrm{R} \leq 30$ & 0,84 & 0,43 \\
\hline $30<\mathrm{R} \leq 40$ & 0,09 & 0,03 \\
\hline $40<\mathrm{R} \leq 50$ & 0,00 & 0,00 \\
\hline $50<\mathrm{R} \leq 60$ & 0,23 & 0,00 \\
\hline $60<\mathrm{R}$ & 0,00 & 0,16 \\
\hline
\end{tabular}

Tabelle 6

Inzuchtkoeffizienten (oben) und deren Verteilung (unten; in \%) (Coefficients of inbreeding (above) and the distribution of them (below; in per cent))

\begin{tabular}{crrrrr}
\hline Inzuchtkoeffizient & Milchkühe & Mutterkühe & Bullen & $\begin{array}{r}\text { Milchvieh- } \\
\text { population }\end{array}$ & $\begin{array}{c}\text { Mutterkuh- } \\
\text { population }\end{array}$ \\
\hline Mittlerer Inzuchtkoeffizient $\bar{F}_{1}(\%)$ & 0,68 & 0,43 & 0,96 & 0,71 & 0,47 \\
Maximaler Inzuchtkoeffizient $\mathrm{F}_{1 \text { max }}(\%)$ & 18,75 & 25,39 & 9,38 & 18,75 & 25,39 \\
Mittlerer Vollständigkeitsindex $\overline{\mathrm{VI}}(\%)$ & 55,15 & 32,48 & 72,92 & 56,96 & 35,84 \\
Mittlerer Inzuchtkoeffizient $\overline{\mathrm{F}}_{2}(\%)$ & 1,24 & 1,34 & 1,32 & 1,25 & 1,32 \\
\hline $\mathrm{F}_{1}=0,000$ & 53,15 & 74,27 & 36,71 & 51,58 & 70,97 \\
$0,001 \leq \mathrm{F}_{1} \leq 1,000$ & 27,08 & 16,05 & 34,18 & 27,80 & 18,29 \\
$1,001 \leq \mathrm{F}_{1} \leq 2,000$ & 11,75 & 4,64 & 16,46 & 12,23 & 5,37 \\
$2,001 \leq \mathrm{F}_{1} \leq 3,000$ & 1,86 & 1,35 & 2,53 & 1,93 & 1,17 \\
$3,001 \leq \mathrm{F}_{1} \leq 4,000$ & 4,15 & 2,13 & 3,80 & 4,12 & 2,01 \\
$4,001 \leq \mathrm{F}_{1} \leq 5,000$ & 0,57 & 0,19 & 2,53 & 0,77 & 0,50 \\
$5,001 \leq \mathrm{F}_{1} \leq 6,000$ & 0,00 & 0,19 & 2,53 & 0,26 & 0,50 \\
$6,001 \leq \mathrm{F}_{1} \leq 7,000$ & 0,57 & 0,39 & 0,00 & 0,51 & 0,34 \\
$7,001 \leq \mathrm{F}_{1} \leq 8,000$ & 0,14 & 0,00 & 0,00 & 0,13 & 0,00 \\
$8,001 \leq \mathrm{F}_{1} \leq 9,000$ & 0,14 & 0,19 & 0,00 & 0,13 & 0,17 \\
$\mathrm{~F}_{1}>9,000$ & 0,57 & 0,58 & 1,27 & 0,64 & 0,67 \\
\hline
\end{tabular}




\subsubsection{Inzuchtkoeffizienten}

Der durchschnittliche Inzuchtkoeffizient $\overline{\mathrm{F}}_{1}$ nimmt bei den Bullen das höchste Ausmaß an, obwohl für sie, im Vergleich mit den Kuhpopulationen, mit Abstand der niedrigste maximale Einzelwert nachgewiesen wurde (Tab. 6). Dementsprechend beläuft sich der Anteil nicht ingezogener Stiere auf nur $37 \%$, während für $51 \%$ der Tiere Inzuchtgrade bis $2 \%$ nachgewiesen wurden. Für die Mutterkühe wurde der geringste durchschnittliche Inzuchtkoeffizient geschätzt, allerdings verknüpft mit weniger mittlerer Vollständigkeit ihrer Stammbäume. Nach Einbeziehung des Vollständigkeitsindex fällt der korrigierte durchschnittliche Inzuchtgrad $\left(\bar{F}_{2}\right)$ im Vergleich zu den anderen Gruppen am höchsten aus.

\subsubsection{Inzuchtrate}

Im Durchschnitt der zurückliegenden Generationen nahm die Inzucht $\left(\Delta \mathrm{F}_{1}\right)$ bei den Bullen am stärksten zu, während ihr Anstieg innerhalb der Milchkühe und insbesondere der Mutterkühe deutlich geringer verlief (Tab. 7). Nach Einbeziehung der Vollständigkeitsindices wandelt sich allerdings das Bild, indem sich die Inzuchtrate $\Delta \mathrm{F}_{2}$ in allen Gruppen fast einheitlich mit 0,31 bzw. 0,33 \% einstellt. Die durchschnittliche Inzuchtrate dürfte wesentlich auf den Inzuchtanstieg von der Elterngeneration zur Generation der aktuellen Zuchttiere zurückzuführen sein, denn dieser wurde sowohl mit $\Delta \mathrm{F}_{3}$ (unkorrigiert) als auch $\Delta \mathrm{F}_{4}$ (korrigiert) in zum Teil bedeutend höherem Ausmaß ermittelt. Vor allem bei den Mutterkühen ragt die Inzucht in der jüngsten Generationsfolge besonders heraus, während sie für die Bullen mit dem geringsten Wert geschätzt wurde. Demgegenüber ist bei den Bullen künftig mit dem höheren Inzuchtanstieg $\left(\Delta \mathrm{F}_{5}\right)$ zu rechnen als bei den übrigen Gruppen.

Tabelle 7

Inzuchtrate (Rate of inbreeding)

\begin{tabular}{cccccc}
\hline Inzuchtrate & Milchkühe & Mutterkühe & Bullen & $\begin{array}{c}\text { Milchvieh- } \\
\text { population }\end{array}$ & $\begin{array}{c}\text { Mutterkuh- } \\
\text { population }\end{array}$ \\
\hline$\Delta \mathrm{F}_{1}$ & 0,17 & 0,11 & 0,24 & 0,18 & 0,12 \\
$\Delta \mathrm{F}_{2}$ & 0,31 & 0,33 & 0,33 & 0,31 & 0,33 \\
$\Delta \mathrm{F}_{3}$ & 0,33 & 0,18 & 0,39 & 0,35 & 0,19 \\
$\Delta \mathrm{F}_{4}$ & 0,57 & 0,73 & 0,44 & 0,57 & 0,69 \\
$\Delta \mathrm{F}_{5}$ & 0,13 & 0,13 & 0,20 & 0,12 & 0,10 \\
\hline
\end{tabular}

4.

Diskussion

\section{Genanteile fremder Populationen}

Der Rasse der Hinterwälder Rinder kann, gemessen am Anteil fremder Rassen, ein hohes Maß an Rassereinheit bescheinigt werden. Diese Eigenschaft kommt insbesondere dann zum Ausdruck, wenn die unbekannten Ahnen vernachlässigt und sie entsprechend der Verteilung der bekannten Herkünfte auf diese umgelegt werden. Diese Vorgehensweise ist zweifelsohne mit Fehlern behaftet, zumal nicht ausgeschlossen werden kann, dass Fremdrassen angehörende Vorfahren vermehrt als unbekannt deklariert wurden, dürfte aber den wahren Verhältnissen in der Population am nächsten kommen.

Der auffallend hohe Anteil unbekannter Herkunft bei den Mutterkühen rührt daher, dass ein Großteil der betreffenden Tiere von Vorfahren abstammt, die ehedem nicht 
als Herdbuchkühe geführt wurden. Tatsächlich sind mit Einführung einer separaten Mutterkuhpopulation etliche Betriebe der Züchtervereinigung beigetreten, so dass zahlreiche neue Tiere ins Zuchtbuch aufgenommen wurden.

Schätzungen von Genanteilen in Rinderpopulationen liegen für mehrere Rassen vor. Für die rheinischen Schwarzbunten (Jahrgang 1983), das württembergische Braunvieh (Jahrgang 1986/87) und die westfälischen Rotbunten (Jahrgang 1984) haben SCHMIDT et al. (1986), BOLLMEIER et al. (1991) und SCHMIDT et al. (1993) die jeweiligen Genanteile der betreffenden Rasse mit 22,4 \%, 52,0 \% bzw. 77,0 \% festgestellt. Die Ergebnisse dürften allerdings durch die nachträgliche Entwicklung der Populationen überholt sein. In jüngeren Untersuchungen von EHLING et al. (1999) anhand der Genreserve Deutscher Schwarzbunter Rinder alter Zuchtrichtung in Niedersachsen wurden Fremdgenanteile (Holstein-Friesian, Jersey) von 0,35 \%, 0,18 \% und 0,49 \% bei 163 Kühen der Institutsherde in Mariensee, 94 Bullen in der Spermabank desselben Instituts bzw. den 38 Bullen mit Spermareserven in verschiedenen Besamungsstationen ermittelt. Die entsprechend hohen Genanteile der Schwarzbunten alten Typs sind somit weitgehend vergleichbar mit den korrigierten Schätzwerten der Hinterwälder Rasse.

\section{Generationsintervalle}

Das Generationsintervall steht im Zusammenhang mit dem Zuchtfortschritt je Zeiteinheit in einer Population. Eine frühere Zuchtbenutzung der Zuchttiere, die Verkürzung der Zwischenkalbezeiten und die Senkung der Nutzungsdauer haben die Verkürzung des Generationsintervalls und damit die Steigerung des Zuchtfortschrittes je Zeiteinheit zur Folge. Eine verminderte Nutzungsdauer verringert jedoch andererseits die mittlere Nachkommenzahl pro Tier, beeinträchtigt die Vermehrungsrate und wirkt somit einer maximalen Selektionsintensität und damit dem Zuchtfortschritt entgegen. Eine Verkürzung der Nutzungsdauer ist deshalb nur dann positiv zu bewerten, wenn das damit verbundene Generationsintervall den Zuchtfortschritt je Zeiteinheit stärker erhöht als dieser durch die reduzierte Selektionsintensität gesenkt wird (SCHÖNMUTH et al., 1986).

Bei Unterstellung von fixen Werten der Selektionsintensität, der Heritabilität, der Wiederholbarkeit bzw. der Genauigkeit der Zuchtwertschätzung hat OLLIVIER (1974) mit dem Ziel eines maximalen Zuchtfortschrittes theoretische Überlegungen über das optimale Abgangsalter von Zuchttieren angestellt. Bei einem Erstkalbealter von zwei bzw. drei Jahren sollte demnach das Alter der Bullen bei Abgang 3,04 bzw. 4,58 Jahre, bei Kühen 6,27 bzw. 8,49 Jahre betragen und die Bestandsergänzung auf der Vaterseite rascher erfolgen als bei den Muttertieren. Damit verbinden sich positive Effekte auf den Zuchterfolg, und zwar einerseits infolge des kürzeren Generationsintervalls der Bullen, die ohnehin hierzu den überwiegenden Beitrag leisten, andererseits infolge der höheren Nachkommenzahl der weiblichen Zuchttiere und damit einer höheren Selektionsintensität innerhalb des weiblichen Geschlechts.

Ähnliche Relationen werden in der Hinterwälder Population angetroffen. Das im Vergleich zu den Kühen zwei bis drei Jahre geringere Durchschnittsalter der Bullen bei der Geburt ihrer männlichen als auch weiblichen Nachkommen lässt sich damit begründen, dass nach wie vor ca. $85 \%$ aller Vatertiere im Natursprung, teilweise als Herden-, teilweise als Gemeindebullen, eingesetzt werden und diese traditionsgemäß 
in der Regel kaum länger als zwei Jahre im Deckeinsatz verbleiben. In der Mutterkuhhaltung ist die Haltung von Herdenbullen ohnehin üblich.

Die das Generationsintervall verlängernde Wartezeit in Verbindung mit künstlicher Besamung und Nachkommenschaftsprüfung entfällt somit für den Großteil der Vatertiere. Aufgrund der Berechnungen von OLLIVIER (1974) müsste sich das optimale Abgangsalter der Bullen unter den Bedingungen der Nachkommenschaftsprüfung auf 10,7 bzw. 12,4 Jahre erhöhen. Damit verbindet sich die Verlängerung der Generationsintervalle auf der Vaterseite.

Dies erklärt die von SCHMIDT (1990) für die westfälische Rotbuntzucht, ENGELHARDT (1996) für die deutsche Schwarzbuntzucht und EHLING et al. (1999) für die Schwarzbunte Genreserve nachgewiesenen deutlich höheren Generationsintervalle der Pfade der Väter zu den Nachkommen im Vergleich zu jenen der Mütter.

Die Bullenmütter zeigen im Mittel ein höheres Alter bei der Geburt der männlichen Nachkommen als die Kuhmütter bei der Geburt ihrer weiblichen Nachkommen. Dies lässt darauf schließen, dass Bullenmütter erst nach Vorliegen von mehr Leistungsinformationen ausgewählt werden, wohingegen weibliche Nachkommen schon von weniger sicheren Müttern nachgezogen werden.

Stellt man die Generationsintervalle Mütter-Nachkommen (5,8-6,8 Jahre) entsprechenden Vergleichswerten, die von SCHMIDT (1990) und ENGELHARDT (1996) bei den Rotbunten (4,2 Jahre) bzw. Schwarzbunten (4,6 Jahre) gefunden wurden, gegenüber, so ist die diesbezügliche Überlegenheit der Hinterwälder unverkennbar. Lediglich für die Genreserve schwarzbunter Rinder alter Zuchtrichtung scheinen die entsprechenden Generationsintervalle nur geringfügig kürzer auszufallen (EHLING et al., 1999).

Die relativ hohen Generationsintervalle Mütter-Nachkommen lassen auf eine relativ lange Nutzungsdauer der Hinterwälder Kühe schließen. Dem Jahresbericht der ADR (2002) kann entnommen werden, dass das Durchschnittsalter der Hinterwälder Herdbuchkühe 6,9 Jahre beträgt, womit sie alle anderen Rassen übertreffen (Murnau-Werdenfelser 6,6 Jahre, Vorderwälder und Pinzgauer jeweils 5,7 Jahre). $21 \%$ aller der vorliegenden Untersuchung zugrunde liegenden Hinterwälder Herdbuchkühe sind älter als 10 Jahre, wobei die derzeit älteste Kuh der aktiven Population nicht weniger als 21 Jahre zählt.

Ungeachtet dessen darf nicht übersehen werden, dass die Generationsintervalle im Laufe der zurückliegenden Generationen rückläufige Tendenz zeigen. Seit der vierten Vorfahrengeneration beträgt ihr Rückgang 0,7 Jahre (Milchkühe) bzw. 0,6 Jahre (Mutterkühe). Dem entspricht der Hinweis von BRODAUF (1995), wonach das Durchschnittsalter der Hinterwälder Herdbuchkühe zwischen 1980 und 1995 von 8 auf 6,8 Jahre gesunken ist. Er begründet diese Entwicklung mit der starken Nachfrage nach weiblichen Zuchttieren (Kühe, Kalbinnen) und dem damit positiv zu bewertenden frühzeitigen Abgang durch Verkauf. Tatsächlich hatten die Hinterwälder in den letzten Jahren regelmäßig etwa ein Drittel aller Abgänge zu Zucht- und Nutzzwecken zu verzeichnen. Bei den Bullen nahm das Generationsintervall bis zur Elterngeneration ebenfalls ab, und zwar von 5,5 auf 4,9 Jahre, um danach zur aktuellen Generation geringfügig um 0,2 Jahre anzuwachsen. Wie gezeigt werden konnte, ist besagter Anstieg nicht bei den männlichen Vorfahren der Bullen zu suchen, sondern auf die Erhöhung des Durchschnittsalters ihrer Mütter um 0,4 Jahre zurückzuführen. Dies deutet darauf 
hin, dass man in jüngerer Zeit zunehmend auf ältere und damit bewährte Kühe als Bullenmütter zurückgreift.

\section{Verwandtschaft}

Die engste verwandtschaftliche Beziehung wurde im Durchschnitt innerhalb der Bullen nachgewiesen. Innerhalb der Milchkühe nehmen sie wesentlich geringere Ausmaße an; am wenigsten scheinen sie innerhalb der Mutterkühe zu existieren. Diese Beobachtung dürfte nicht zuletzt mit den erheblichen Unterschieden hinsichtlich der Vollständigkeit der Stammbäume in den drei Tiergruppen in Verbindung stehen. Die sehr geringe Vollständigkeit bei den Mutterkühen steht damit im Zusammenhang, dass weibliche Vorfahren zahlreicher Mutterkühe vielfach nicht im Herdbuch registriert waren.

Von geringen Abweichungen abgesehen, haben SCHMIDT et al. (1986) für die rheinischen Schwarzbunten, BOLLMEIER et al. (1991) für das württembergische Braunvieh und JAGUSIAK und ZARNECKI (1994) für die Schwarzbunten in Polen vergleichbare Verwandtschaftsgrade der von ihnen untersuchten Populationen finden können, wobei, wie in der vorliegenden Analyse, die engeren Bindungen innerhalb der Bullen nachgewiesen wurden. Anders verhält es sich offenbar bei der schwarzbunten Genreserve Niedersachsens, bei der gegenteilige Verhältnisse vorliegen.

Die unterschiedliche Vollständigkeit der Pedigrees bei den Milch- und Mutterkühen macht sich auch hinsichtlich ihrer mittleren Verwandtschaft zu den Vatertieren bemerkbar. Dementsprechend fällt der mittlere Verwandtschaftsgrad der Mutterkühe zu den Bullen geringer aus (1,4\%), als dies für die Milchkühe zutrifft (2,6 \%). Zumindest für letztere wird gleichzeitig eine engere verwandtschaftliche Beziehung zu den Bullen festgestellt, als sie SCHMIDT et al. (1986) bei den rheinischen Schwarzbunten mit 1,0 $\%$ bzw. 1,2 \% nachgewiesen haben.

\section{Inzucht}

Insbesondere in kleinen Populationen verdient die Inzucht infolge Verminderung der genetisch nutzbaren Varianz und damit des Zuchtfortschrittes sowie erhöhter Gefahr der Entstehung von Depressionen bzw. Erbdefekten vermehrt Aufmerksamkeit. Bei Berücksichtigung von fünf Ahnengenerationen und nach Korrektur mittels Pedigreevollständigkeit resultierte der durchschnittliche Inzuchtgrad für alle Teilpopulationen mit ca. $1,3 \%$.

Dieses Ergebnis lässt sich im Rahmen der von KROGMEIER et al. (1997) für das Gelb- und Braunvieh und EHLING et al. (1999) für die Genreserve schwarzbunter Rinder alter Zuchtrichtung einordnen. Hingegen scheint die durchschnittliche Inzucht bei den rheinischen Schwarzbunten (SCHMIDT et al., 1986), beim württembergischen Braunvieh (BOLLMEIER et al., 1991) und bei den westfälischen Rotbunten (SCHMIDT et al., 1993) mit Durchschnittskoeffizienten unter 1,0 \% wesentlich geringer zu sein. Allerdings darf dabei nicht übersehen werden, dass besagte Untersuchungen teilweise mehrere Jahre zurückliegen und dem aktuellen Stand kaum mehr entsprechen dürften. Auch ausländische Analysen, wie die von HAGGER (1988) am schweizerischen Braunvieh, MIGLIOR et al. (1990) an kanadischen Jersey- und Holstein-Kühen, NAVAJAS und URIOSTE (1995) an Aberdeen Angus-Kühen in Argentinien, YOUNG und SEYKORA (1996) am US-amerikanischen Holstein-Kühen und ROUGHSEDGE et al. (1999) an Holstein Friesian-Kühen Großbritanniens gelangten 
zu Inzuchtkoeffizienten, die auf relativ geringem Niveau (0,4-0,8 \%) angesiedelt sind. Dabei macht sich bemerkbar, dass diese für große Populationen gelten.

Inwieweit die Inzucht der Hinterwälder Population als bedenklich einzustufen ist, lässt sich kaum abschätzen. Die Wertung von KROGMEIER et al. (1997) und EHLING et al. (1999), deren Schätzwerte von ähnlichem Ausmaß sind und die daraus folgern, dass die Inzucht als moderat einzustufen sei, kann auch für die Hinterwälder in Anspruch genommen werden.

Damit im Zusammenhang ist die von der effektiven Populationsgröße abhängige Inzuchtrate zu sehen. Von der DGfZ wurde 1992 die Empfehlung ausgesprochen, dass die Inzuchtsteigerung je Generation 1 \% nicht übersteigen sollte, was bei Zufallspaarung einer effektiven Populationsgröße von $\mathrm{N}_{\mathrm{e}}=50$ entspricht. Das inzuchtbedingte Schadensrisiko scheint sich entsprechend allgemeiner Erfahrung bis zu diesem Schwellenwert in verkraftbaren Grenzen zu halten, so dass sich damit die Voraussetzung für den langfristigen Erhalt der Population verbinden dürfte.

Wie sich anhand der Inzuchtraten seit der fünften Ahnengeneration einerseits und in der letzten Generation andererseits abzeichnet, hat in den zurückliegenden Zuchtphasen eine Zunahme der Inzuchtsteigerung stattgefunden. Dennoch scheint sich die Hinterwälder Population derzeit noch weit entfernt von besagter Gefahrenzone zu entwickeln. Möglicherweise wird bewusst auf die Paarung nicht oder wenig verwandter Tiere Wert gelegt. Unter Nutzung des großen Anteils der Bullen, die mit den Kühen nicht oder nur wenig verwandt sind, gilt es auch in Zukunft darauf zu achten. Besonders gering fällt der zu erwartende Inzuchtanstieg aufgrund der aktuellen effektiven Väter- und Mütterzahl aus. Dazu trägt vor allem die in jüngerer Vergangenheit gestiegene Zahl an Vatertieren bei.

In diesem Zusammenhang macht PIRCHNER (2002) darauf aufmerksam, dass in Doppelnutzungsrassen mit einer relativ größeren Anzahl eingesetzter Vatertiere gerechnet werden kann als in Milchrassen, da ein Teil wegen des hohen Milchzuchtwertes, ein anderer wegen guter Fleischleistung bei akzeptablem Milchzuchtwert selektiert wird. Dementsprechend stellte er anhand von Markergenfrequenzen den Inzuchtzuwachs beim bayerischen Fleckvieh trotz hoher KB-Anteile innerhalb von 6,25 Generationen mit nur 0,13 bzw. 0,16 \% fest.

Dem Doppelnutzungsstatus dürften trotz geringer Populationsgröße die dem Inzuchtzuwachs der Hinterwälder sehr ähnlichen Inzuchtraten der von EHLING et al. (1999) untersuchten niedersächsischen Genreserve der Schwarzbunten alter Zuchtrichtung zu verdanken sein, und zwar sowohl in zurückliegenden Generationen als auch hinsichtlich des in Zukunft zu erwartenden Inzuchtanstieges. Die von PIRCHNER (2002) für die ebenfalls sehr kleine Population des Tiroler Grauviehs innerhalb von 5,8 Generationen nachgewiesene Inzuchtrate ist mit 0,51 \% zwar etwas höher und wird mit der in den letzten Jahrzehnten verstärkten Selektion auf Milchleistung begründet. Demgegenüber zeichnet sich für die Murnau-Werdenfelser Population anhand der von Markergenfrequenzen errechneten Inzuchtrate eine bedenkliche Situation ab (GRAML et al., 1988); bezogen auf den Zeitraum von 4,6 Generationen wurde ein Inzuchtanstieg von 1,43 \% ermittelt, für die Zukunft wurde ein solcher von sogar 2,35 \% prognostiziert.

Einzelne sehr hohe Inzuchtkoeffizienten haben zwar keinen bedeutenden Einfluss auf den mittleren Inzuchtgrad der Population, können aber in Einzelfällen bedenklich sein, auch wenn dramatische Einbrüche durch Inzuchtdepressionen offensichtlich nicht zu 
erwarten sind (HAGGER, 1988; CASANOVA et al., 1992; MIGLIOR et al., 1992; BURROW, 1993; NAVAJAS und URIOSTE, 1995; KROGMEIER et al., 1997; SMITH et al., 1998; THOMPSON et al., 2000). Langfristig wird eine möglicherweise kritische Inzuchthöhe nicht vermeidbar sein. Deshalb ist die Beibehaltung der angelegten Spermareserven von Hinterwälder Bullen (SIMON und BUCHENAUER, 1993) als sicherheitsbildende Maßnahme von hohem Wert. Sie sollte zweckmäßigerweise von Zeit zu Zeit durch Samen jüngerer, unverwandter Bullen aktualisiert werden. Schließlich kann im Bedarfsfall auf die züchterische Zusammenarbeit mit der schweizerischen Hinterwälder Population zurückgegriffen werden. Vorerst besteht hierzu jedoch keine Notwendigkeit.

\section{Danksagung}

Der Rinder-Union Baden-Württemberg wird für die Bereitstellung der Zuchtbuchauszüge herzlich gedankt.

\section{Literatur}

ADR:

Rinderproduktion in der Bundesrepublik Deutschland 2001. (2002)

BOLLMEIER, S.; MAYER, M.; SIMON, D.:

Entwicklung der genetischen Struktur beim württembergischen Braunvieh. Züchtungskunde 63 (1991), 1-11 BRODAUF, W.:

Bodenständige Haustierrassen des Schwarzwaldes - Entstehung, Bestand und Gründe für die Erhaltung. Mitt. Badischer Landesvereine für Naturkunde und Naturschutz. N.F. 16 (1995), 297-317

BURROW, H.M.:

The effects of inbreeding in beef cattle. Anim. Breeding Abstr. 61 (1993), 737-751

CASANOVA, L.; HAGGER, C.; KÜNZI, N.; SCHNEEBERGER, M.:

Inbreeding in Swiss Braunvieh and its influence on breeding values predicted from a repeatability animal model. J. Dairy Sci. 75 (1992), 1119-1126

DGfZ:

Empfehlungen zur Erhaltung lebender Tierbestände einheimischer gefährdeter Nutztierrassen. Züchtungskunde 62, (1992), 77-80

EHLING, C.; SCHMIDT, T.; NIEMANN, H.:

Untersuchungen zur genetischen Struktur und Diversität der Genreserve Deutscher Schwarzbunter Rinder alter Zuchtrichtung. Züchtungskunde 71 (1999), 130-146

ENGELHARDT, I.:

Inzucht, bedeutende Ahnen und Wahrscheinlichkeit für BLAD-Merkmalsträger in der Deutschen Schwarzbuntzucht. Hannover, Diss., 1996

GRAML, R; LANG, G; SCHMID, D.O; WITTKOWSKI, G.; KRAUSE, I; BUCHBERGER, J; PIRCHNER, F.: Schätzung von Inzuchtzuwachs und effektiver Populationsgröße des Murnau-Werdenfelser Rindes und Deutschen Fleckviehs aus Markergenhäufigkeiten. Züchtungskunde 60 (1988), 288-299

HAGGER, C.:

Stand der Inzucht im Schweizer Braunvieh. Landwirtschaft Schweiz 1 (1988), 293-296

JAGUSIAK, W.; ZARNECKI, A.:

Relationship and inbreeding among black-and-white bulls used in artificial insemination. Prace I Materialy Zootechniczne 46 (1994),75-81

KÖTTERITSZCH, O.; MAUS, F.:

110 Jahre Hinterwälderzucht. Festschrift (1999)

KROGMEIER, D.; AUMANN, J.; AVERDUNK, G.:

Untersuchungen zur Inzucht in der Gelbvieh- und Braunviehpopulation in Süddeutschland. Züchtungskunde 69 (1997), 233-243

LKV Baden-Württemberg:

Jahresberichte des Landesverbandes Baden-Württemberg für Leistungsprüfungen in der Tierzucht. (diverse Jahrgänge)

MAUS, F.:

Die Wälderrassen. Landinfo H. 5 (1997)

MIGLIOR, F.; BURNSIDE, E.B.; SULLIVAN, B.P.; SZKOTNICKI, B.; KENNEDY, B.W.: 
Trends in inbreeding in Canadian dairy cattle. Proc. $4^{\text {th }}$ World Congress on Genetics applied to Livestock Prod., Edinburgh (1990), 183-186

MIGLIOR, F.; SZKOTNICKI, B.; BURNSIDE, E.B.:

Analysis of levels of inbreeding and inbreeding depression in Jersey cattle. J. Dairy Sci. 75 (1992), 11121118

NAVAJAS, E.A.; URIOSTE, J.I.:

Effects of inbreeding and preweaning growth of Aberdeen-Angus calves. Revista Argentina de Produccion Animal 15 (1995), 880-883

OLLIVIER, L.:

Optimum replacement rates in animal breeding. Anim. Prod. 19 (1974), 257-271

PIRCHNER, F.:

Schätzung inzuchtwirksamer (effektiver) Populationsgrößen aus Genfrequenzschwankungen bei Bayerischem Fleckvieh und Tiroler Grauvieh. Arch. Tierz., Dummerstorf 45 (2002), 331-339

ROUGHSEDGE, T.; BROTHERSTONE, S.; VISSCHER, P.M.; BOYAZOGLU, J.; RAFAI, P., THOMAS, C.;

ZJALIC, M.:

Quantifying genetic contributions to a dairy cattle population using pedigree analysis. EAAP Publication 60 (1999), 359-369

SCHMIDT, T.:

Analyse der westfälischen Rotbuntzucht bezüglich Inzucht, Verwandtschaft, Fremdgenanteil, Generationsintervall und Zuchtfortschritt. Univ. Bonn, Diss., 1990

SCHMIDT, T.:

OPTI-MATE Vers. 3.3. Ein Management-Programm zur Minimierung der Inzucht in gefährdeten Populationen. Programmbeschreibung (2000)

SCHMIDT, T.; MAYER, M.; SIMON, D.:

Analyse der westfälischen Rotbuntzucht bezüglich Inzucht, Verwandtschaft und Fremdgenanteil. Züchtungskunde 65 (1993), 102-111

SCHMIDT, T.; MAYER, M.; SIMON, D.:

Inzucht, Verwandtschaft und Genanteile in der rheinischen Schwarzbuntzucht. Vortragstagung der DGfZ und GfT (1986)

SCHÖNMUTH, G.; FLADE, D.; SEELAND, G.:

Züchterische und ökologische Grundlagen. Verlag Harri Deutsch, Thun, Frankfurt/Main (1986)

SIMON, D.L.; BUCHENAUER, D.:

Genetic diversity on European livestock breeds. EAAP Publication Nr.66, Wageningen (1993)

SMITH, L.A.; CASSELL, B.G.; PEARSON, R.E.:

The effect of inbreeding on the lifetime performance of dairy cattle. J. Dairy Sci. 81 (1998), 2729-2737

THOMPSON, J.R.; EVERETT, R.W.; HAMMERSCHMIDT, N.L.:

Effects of inbreeding on production and survival in Holsteins. J. Dairy Sci. 83 (2000), 1856-1864

WIGGANS, G.R.; VAN RADEN, P.M.; ZUURBIER, J.:

Calculation and use of inbreeding coefficients for genetic evaluation of United States dairy cattle. J. Dairy Sci. 78 (1995), 1584-1590

WRIGHT, S.:

Mendelian analysis of the pure breeds of livestock. 1. The measurement of inbreeding and relationship. J. Hered. 14 (1923), 339-348

YOUNG, C.W.; SEYKORA, A.J.:

Estimates of inbreeding and relationship among registered Holstein females in the United States. J. Dairy Sci. 79 (1996), 502-505

Eingegangen: 05.03.2003

Akzeptiert: 27.05.2003

Anschriften der Verfasser

Prof. Dr. GÜNTER BIEDERMANN

Dipl.-Ing. SUSANNE WALDMANN

Fachgebiet Tierzucht, Universität Kassel

Nordbahnhofstraße 1a

D-37213 Witzenhausen
Dr. FRANZ MAUS

Referat Tierzucht

ALLB Donaueschingen

Irmastraße 3

D-78166 Donaueschingen 